\section{The devastating power of platelets in COPD exacerbations: can aspirin save lives in COPD?}

\author{
Don $D \operatorname{Sin}^{1,2}$
}

The human and societal burden of COPD is alarming. Despite large reductions in smoking rates across industrialised nations, COPD mortality has increased by $60 \%$ over the past 20 years, making it the second leading cause of morbidity and mortality in the USA and elsewhere. ${ }^{1}$ Most of the COPD-related deaths occur during or shortly following acute exacerbations (or 'lung attacks'). Unfortunately, severe lung attacks are common, with one in six patients requiring hospitalisation for urgent care each year. ${ }^{2}$ Despite best therapy, 1 in 12 of these patients will succumb to their disease in hospital. ${ }^{3}$ Even those who survive will experience persistent morbidity from their recent lung attack and never regain their lost health status. Furthermore, one in three patients will have another lung attack in 6-12 months. ${ }^{3}$ Regrettably, acute and chronic treatments for COPD are suboptimal. Despite global drug expenditures that exceed $\$ 36$ billion annually for COPD care, ${ }^{4}$ none of the currently available drugs reduces mortality or modifies disease progression as defined by rate of decline in lung function. ${ }^{5}$ Although the existing drugs produce symptomatic benefits and ameliorate exacerbations, the effect is very modest, reducing the risk of exacerbations by only $10-25 \%$, even in multiple combinations. ${ }^{5}$ Drug discovery has been slow and largely disappointing, largely owing to poor understanding of disease pathobiology, inadequate phenotyping, suboptimal use of animal models and the high cost (and risk) of drug development.

To address these gaps, there has been a growing interest in developing simple blood-based biomarkers of disease activity in COPD. Although there has been tremendous progress in this area over the past decade, there are no commercially

\footnotetext{
${ }^{1}$ UBC James Hogg Research Center and The Institute for Heart and Lung Health, St Paul's Hospital, Vancouver, British Columbia, Canada; ${ }^{2}$ Department of Medicine (Pulmonary Division), University of British Columbia, Vancouver, British Columbia, Canada

Correspondence to Don D. Sin, St Paul's Hospital, 1081 Burrard Street, Vancouver, British Columbia, Canada V6Z 1Y6; don.sin@hli.ubc.ca
}

available blood tests that can be used for this purpose and it will take years before promising novel biomarkers make it to the clinics. The paper by Harrison and colleagues in this issue of Thorax offers a new approach to biomarker and therapeutic discovery that may enable more rapid clinical translation. ${ }^{6}$ Instead of relying on discovery of de novo protein or genomic signatures, Harrison and colleagues assessed the prognostic value of blood platelet count, which is widely available, cheap and well standardised, during acute lung attacks of COPD. In this large, well conducted cohort study of 1343 patients, who were hospitalised for an exacerbation, they found that thrombocytosis defined as a blood platelet count of $>400 \times 10^{9}$ cells $/ \mathrm{mm}^{3}$ at admission was associated with a $137 \%$ increase in the risk of in-hospital mortality and a $53 \%$ increase in 1-year mortality. Most importantly, they observed that treatment with an anti-platelet drug such as aspirin or clopidogrel was associated with a threefold reduction in the 1-year mortality rate. ${ }^{6}$ Interestingly, none of this appeared to be directly related to a reduction in cardiovascular risk, suggesting mechanisms beyond the salutary effects on the cardiovascular system.

The sceptics will argue that these results cannot be taken seriously as they were not generated from a high-quality randomised controlled trial and thus could be confounded by a host of different measured and unmeasured factors. However, before dismissing them, it is essential to consider the biological plausibility of the reported findings. Although the traditional function of platelets is to 'plug' and 'seal' haemostatic wounds, the modern view is that they perform a variety of different and complex functions that impact on the pathogenesis of diseases such as cancer, sepsis, rheumatoid arthritis and even malaria. ${ }^{7-9}$ The critical step in this process is platelet activation, which causes these cells to unleash a cascade of chemokines, cytokines, growth factors, and other molecules that promote inflammation, tissue injury, coagulation, and even cell death. Patients with COPD demonstrate increased platelet activation, with further activation occurring during acute exacerbations. ${ }^{10}$ The activated platelets in turn amplify the inflammatory signal and promote clotting and effect tissue damage. Clopidogrel inhibits platelet activation by irreversibly binding to $\mathrm{P}_{2} \mathrm{Y}_{12}$ receptor on platelet cell membranes. Aspirin, however, irreversibly inhibits cyclooxgenase enzymes and prevents the formation of thromboxane and other prostaglandins in platelets, which in turn confers cardiovascular protection and prevents death from colorectal cancer. ${ }^{11}$ These drugs have been tested in large numbers of individuals and have been shown to have an acceptable safety profile.

Should patients with COPD be treated with an anti-platelet agent during acute or following exacerbations? Unless there is a medical contraindication, low-dose aspirin (ie, $80-100 \mathrm{mg} / \mathrm{day}$ ) appears to be a reasonable therapeutic choice for most patients with COPD in view of their increased risk of cardiovascular disease and colorectal cancer. ${ }^{12}$ The data reported by Harrison and colleagues also suggest that there may be a low-cost solution to finding 'novel' biomarkers in COPD by re-purposing existing blood tests for patients with COPD. ${ }^{6}$ This approach may accelerate biomarker discovery and most importantly lead to novel therapeutic targets, which are urgently needed to confront the growing worldwide burden of COPD that is taking the breath away from hundreds of millions of patients each year.

Competing interests None.

Provenance and peer review Not commissioned; internally peer reviewed.

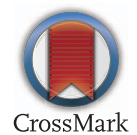

To cite Sin DD. Thorax 2014;69:603-604.

Received 24 February 2014

Accepted 7 March 2014

Published Online First 17 April 2014

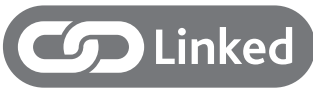

http://dx.doi.org/10.1136/thoraxjnl-2013-203996

Thorax 2014;69:603-604.

doi:10.1136/thoraxjnl-2014-205329

\section{REFERENCES}

1 US Burden of Disease Collaborators. The state of US health, 1990-2010: burden of diseases, injuries, and risk factors. JAMA 2013:310:591-608.

2 Hurst JR, Vestbo J, Anzueto A, et al. Susceptibility to exacerbation in chronic obstructive pulmonary disease. N Engl J Med 2010;363:1128-38.

3 Leuppi JD, Schuetz P, Bingisser R, et al. Short-term vs conventional glucocorticoid therapy in acute 
exacerbations of chronic obstructive pulmonary disease: the REDUCE randomized clinical trial. JAMA 2013:309:2223-31.

4 IMS Institute for Health Informatics. The Global Use of Medicines: Outlook Through 2015. http://www. imshealth.com/deployedfiles/ims/Global/Content/ Insights/IMS\%20Institute $\% 20$ for $\% 20$ Healthcare $\%$ 20Informatics/Global_Use_of_Medicines_Report.pdf (accessed 18 Feb 2014).

5 Vestbo J, Hurd SS, Agusti AG, et al. Global strategy for the diagnosis, management, and prevention of chronic obstructive pulmonary disease: GOLD executive summary. Am J Respir Crit Care Med 2013;187:347-65.
6 Harrison M, Short P, Williamson P, et al. Thrombocytosis is associated with increased short and long term mortality after exacerbation of chronic obstructive pulmonary disease: a role for anti-platelet therapy? Thorax 2014;69: 609-15.

7 Boilard E, Nigrovic PA, Larabee K, et al. Platelets amplify inflammation in arthritis via collagendependent microparticle production. Science 2010;327: 580-3.

8 Grewal PK, Uchiyama S, Ditto D, et al. The Ashwell receptor mitigates the lethal coagulopathy of sepsis. Nat Med 2008;14:648-55.
9 Ho-Tin-Noe B, Goerge T, Cifuni SM, et al. Platelet granule secretion continuously prevents intratumor hemorrhage. Cancer Res 2008;68:6851-8.

10 Maclay JD, McAllister DA, Johnston S, et al. Increased platelet activation in patients with stable and acute exacerbation of COPD. Thorax 2011:66:769-74.

11 Davi G, Patrono C. Platelet activation and atherothrombosis. N Engl J Med 2007;357:2482-94.

12 Anthonisen NR, Connett JE, Enright PL, et al. Lung Health Study Research Group. Hospitalizations and mortality in the Lung Health Study. Am J Respir Crit Care Med 2002;166:333-9. 